\title{
Analysis of Network Parameters Influencing Performance of Hybrid Multimedia Networks
}

\author{
Pavel Masek ${ }^{1}$, Dominik Kovac ${ }^{2}$, Jiri Hosek ${ }^{3}$, Mariya Pavlova ${ }^{4}$, Ondrej Krajsa ${ }^{5}$
}

\begin{abstract}
Multimedia networks is an emerging subject that currently attracts the attention of research and industrial communities. This environment provides new entertainment services and business opportunities merged with all wellknown network services like VoIP calls or file transfers. Such a heterogeneous system has to be able to satisfy all network and end-user requirements which are increasing constantly. Therefore, there is a need for simulation tools enabling deep analysis to find key performance indicators and factors which influence the overall quality for specific network service. This paper provides a study on the network parameters like communication technology, routing protocol, QoS mechanism, etc. and their effect on the performance of hybrid multimedia network. The analysis was performed in OPNET Modeler environment and the most interesting results are discussed at the end of this paper.
\end{abstract}

Keywords - Multimedia networks, Network KPI, OPNET Modeler, Performance analysis, PON, QoS, Routing system.

\section{INTRODUCTION}

The most recent trend in the field of communication networks clearly leads to a convergence of all types of services under a single communication platform known as a hybrid multimedia network. Such a network must be able to efficiently handle traditional voice calls, emerging real-time videoconferencing applications or generic data which consist of a large group of network services (email, web, remote access, etc.). Moreover, end-users demands for high quality of network services are constantly growing over the time. Therefore, multimedia networks must be able to meet all strict performance criteria.

Even though modern multimedia networks differ in many parameters, i.e. the topology, type of provided services, link utilization, used communication technologies, etc., they are mostly built on TCP/IP platform which provides a number of advantages already discussed many times. On the other hand, general architecture presents a number of interfaces or subsystems that can potentially affect the network KPIs (Key Performance Indicators), e.g. throughput, end-to-end delay, jitter, etc.). One of the most important factors that significantly affect network KPIs and thus the overall

Manuscript received November 28, 2013. This work has been supported by the project $n$. FR-TI4/580.

Pavel Masek ${ }^{1}$, Dominik Kovac ${ }^{2}$, Jiri Hosek $^{3}$ and Ondrej Krajsa ${ }^{5}$ are with Department of Telecommunications, Brno University of Technology, Brno, Czech Republic (e-mails: xmasek12@phd.feec.vutbr.cz ${ }^{1}$, xkovac23@phd.feec.vutbr.cz ${ }^{2}, \quad \underline{\text { hosek@feec.vutbr.cz }}{ }^{3}$, krajsao@feec.vutbr.cz ${ }^{5}$ ).

Mariya Pavlova ${ }^{4}$ is with the Department of Communication Networks and Telecommunication Systems, Kalashnikov Izhevsk State Technical University, Izhevsk, Russia (e-mail: marya.m.pavlova@gmail.com ${ }^{4}$ ). quality of service perceived by end-users is the routing subsystem. However, it is not just about choosing the most suitable routing protocol.

Especially for the communication networks operating the multimedia data transmission in real-time, complementary mechanisms and algorithms closely related to the routing protocol are very important. These mechanisms include methods to ensure QoS (Quality of Service) which aims to provide preferential treatment for specific type of network over others. Due to this fact, it is possible to achieve the desired KPIs for various types of multimedia services.

When building a communication network or implementing new services, it is always very important to perform a thorough analysis and testing before production operation. Testing in a real network is in most cases very expensive and therefore variety of network simulators and emulators are used to verify the correct functioning of newly developed technologies and protocols.

In this work, the analysis and testing of routing subsystem and related mechanisms in order to provide maximal performance of hybrid multimedia network is performed. For this purpose, several simulation scenarios were developed in the OPNET Modeler environment.

\section{Optimization OF NETWORK TECHNIQUES FOR HYBRID MULTIMEDIA NETWORKS}

With the gradual development of applications working in real-time, the end-users demands for compliance with predefined parameters during data transfer through network infrastructure grow. The most important of KPIs, also recognized as QoS parameters, are the end-to-end delay, jitter or network throughput. Specific values of these QoS parameters are needed to be ensured during data transfers carried out in the hybrid multimedia network to provide demanded quality perceived by end-user.

To achieve excellent KPIs in multimedia networks, various methods, algorithms and technologies are available. This study includes the evaluation of some of these methods, like the optimization of routing protocol parameters, the classification of network traffic into VLANs (Virtual Local Network) or an implementation of QoS mechanism.

\section{A. Routing protocols in multimedia networks}

Selecting a suitable routing protocol and precise setting of its parameters is very important to get an efficient data transmission in the network.

The routing is defined as a process that performs special routing devices called routers to find the most efficient route to deliver the packet to the destination node along this route. The routers are governed by destination IP address which they read out from the IP packet header. To make their 
decision about next packet routing accurate and correct, the routers must learn about routes to other networks. There are two methods how the router can learn a new route to other networks: statically and dynamically [1].

The static routing is typically used in small networks or in networks where the topology changes are not so frequent. It is therefore obvious that for the hybrid multimedia networks, the dynamic routing must be used. In case of dynamic routing, the information sharing between routers is realized through a routing protocol. The classification of dynamic routing protocols is shown in Fig. 1.

The information exchanged among routers (including the distance to other networks) enables to create and update routing tables on every router in the network [1].

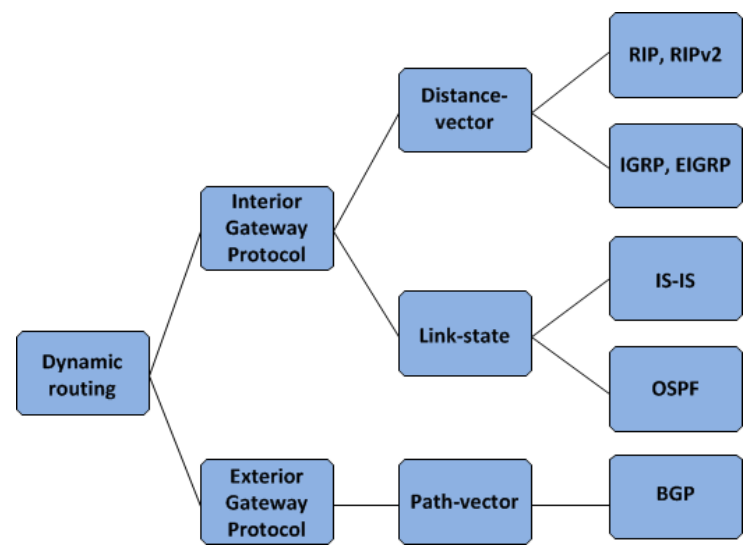

Fig. 1. Dynamic routing protocols

The essential part of the dynamic routing is the routing algorithm. According to the used routing algorithm, the routing protocols are divided into two large groups:

- Distance-vector protocols: These protocols are also referred to as Bellman-Ford or Ford-Fulkerson routing protocols.

- Link-state protocols: These routing protocols generate complete network map called Link State Database. On the top of this database, the best route is calculated by the help of SPF (Shortest Path First) algorithm.

In this paper, we are focusing on two the most famous routing protocols for local networks - RIP (Routing Information Protocol) and OSPF (Open Shortest Path First) to satisfy the requirements of hybrid multimedia networks.

\section{1) RIP (Routing Information Protocol)}

The RIP (Routing Information Protocol) protocol belongs to the class of routing protocols which use a distance vector as a key parameter. The operation of RIP is based on the periodic exchange (so-called triggered updates) of complete routing tables between neighboring routers. The exchange is performed every 30 seconds or when the network topology is changed.

A router that detects a change sends immediately the triggered update to the neighboring routers and this information about the topology changes is disseminated further [2]. There are two versions of the RIP protocol. The so-called RIPv1 is formally defined in RFC 1058 [3]. The second expanded version, RIPv2, is described in RFC 2453 [4].

\section{2) OSPF (Open Shortest Path First)}

The OSPF (Open Shortest Path First) protocol is an open protocol which is more suitable for larger networks [5]. Its specification is available as RFC (Request For Comments). The first version of OSPF is described in RFC 1131 [6]. The second version (OPSFv2) is described in RFC 2328 [7]. Within the introduction of IPv6 (Internet Protocol version 6), the third version (OSPFv3) was defined in RFC 2740 [8]

The functional principle of OSPF protocol is based on the flood-based dissemination of LSA (Link State Agreement) messages which contain information about the networks to which the router is connected [2].

\section{B. Quality of Service in hybrid multimedia networks}

The requirement for ensuring demanded service quality is directly related to the concept of QoS which means an effort to comply with predefined parameters during the data transfer. QoS assurance is very important, especially, for VoIP or videoconferencing services which are strongly time-sensitive and their popularity is still growing.

There are different solutions and network architectures that provide a classification of network traffic into certain classes with specific priority. Quality of service can be implemented in different network layers. The most commonly used QoS implementations are working on datalink and/or network layer [9].

The data-link layer QoS solutions are connected with the ATM (Asynchronous Transfer Mode) or Ethernet technologies. Considering the QoS implementation on network layer, there are two well-known mechanisms Integrated Services (IntServ) and Differentiated Services (DiffServ) [10].

Since the IntServ has several practical limitations, it has never been widely implemented. Therefore, currently the most successful QoS technology in IP networks is the DiffServ. One of the fundamental characteristics of DiffServ mechanism is that all network traffic processing, which includes classifying and measuring the intensity of the network stream, packet marking and sorting and packet forwarding, is made at the edge routers [11]. Fundamental principle of DiffServ mechanism, where individual data flows are aggregated into several classes based on the service type, is shown in Fig. 2.

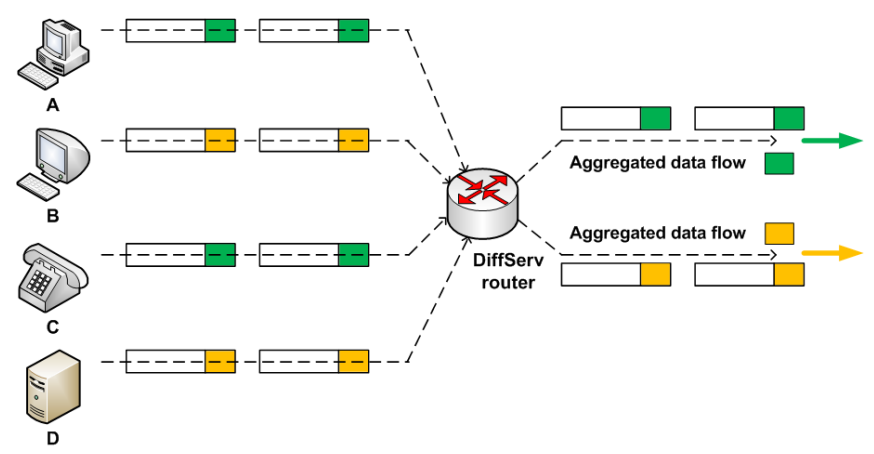

Fig. 2. Aggregation of network flows within DiffServ technology

Each packet entering the network is signed by a special mark - DSCP (DiffServ Code Point) stored in IP header, which expresses how other routers should process each specific packet. This marking process is performed only at 
the network entrance (edge router) [12].

The DSCP identifier is sufficient to classify network traffic into classes, so there is no need for a signaling protocol to create a reservation status, as the IntServ mechanism does. Furthermore, since the traffic is aggregated and then processed within classes, the need for a complex sorting and planning of network resources for the individual data streams is eliminated $[13,14]$.

The simplicity and low overloading of network devices are among main advantages of DiffServ [15] mechanism and also a reason why this technology has been quite popular so far [16].

\section{Virtual Local Area Networks}

Another way how to classify network traffic is by the help of VLAN (Virtual Local Area Network). Virtual network is a group of network devices that are arbitrarily interconnected in computer networks and behave as if they are on the same physical medium which is separated from the rest of network $[17,18]$. The VLAN concept is shown in Fig. 3.

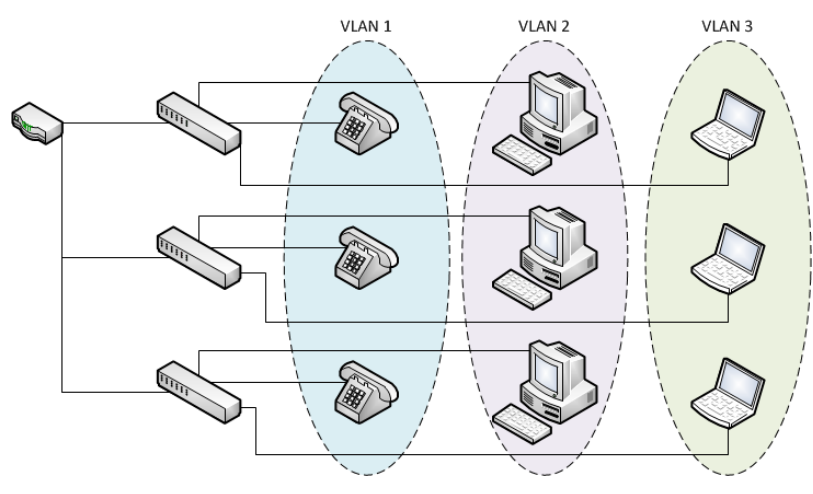

Fig. 3. Network traffic classification into VLANs

Virtual networks allow to interconnect the remote devices and separate these devices from others virtual networks at the same time. The LAN segments where the broadcast traffic is spread are not limited by physical connection to the router but may be defined arbitrarily as needed. Virtual networks can divide a large network into logical units that act as independent local networks. The segmentation of networks is necessary for the limitation of the broadcast domain and also because of security and administrative reasons. A suitably divided network is easier to handle the amount of traffic and network load [17, 18].

Each virtual network is defined by the identifier (so called color). The packets may be transmitted from one segment to another only if the packet contains of the same identifier. The great advantage of VLANs is a possibility to change the logical network topology without any need to modify the physical position of the devices (routers, switches) or their addresses. The segmentation of LANs into multiple logical segments can be performed according to their functionalities, user type, operated services etc.

The VLAN identifier (tag) is attached to Ethernet frame. The tag is used by a destination device for the detection if the source device belongs to the same VLAN network.

The quality of service in VLAN networks is provided through the QoS Match VLAN mechanism. This mechanism allows to split and to prioritize the network traffic based on VLAN identifier. E.g. individual VLAN can be created for
VoIP or VoD service and prioritize these VLANs. The prioritization of selected VLANs guarantees that required quality of service is assured [18]. The most commonly used VLAN standard is the tagging protocol IEEE 802.1Q (see the structure IEEE 802.1Q frame in Fig. 4) [19].

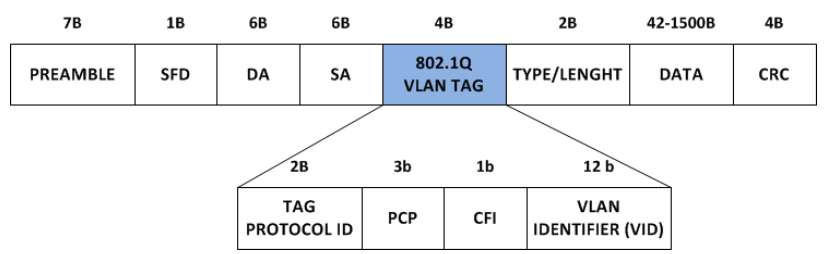

Fig. 4. Structure of framework IEEE 802.1Q

\section{SiMULATION MODEL}

To verify the influence of the transmission technologies, routing protocols and QoS mechanisms on the performance of the multimedia network and the final quality of the service provided $_{2}$ the OPNET Modeler ver. 17.5 simulation environment was chosen.

\section{A. Simulation of $P O N$}

PON (Passive Optical Network) is one of communications technologies which has very good performance characteristics and thus is a good candidate for hybrid multimedia networks. Even though the costs for PON's deployment are higher in comparison with traditional IP networks, the popularity of these networks is increasing rapidly [20].

The main goal of the simulation of passive optical network was to verify the ability to serve as a communication platform for hybrid multimedia networks. The demonstration was compiled in a single passive optical network. The network model developed in OPNET Modeler (see Fig. 5) was built with respect to a logical context. Therefore, it does not reflect real distances between nodes. It was based on a simple tree topology where the network consists of one OLT (Optical Line Termination), one passive optical splitter and two ONUs (Optical Terminal Units).

Network nodes are interconnected with an optical link with maximal throughput $1 \mathrm{Gbps}$. The parameters of data sources connected to the ONUs were varied during the simulation as needed. However, the access network topology remained the same.

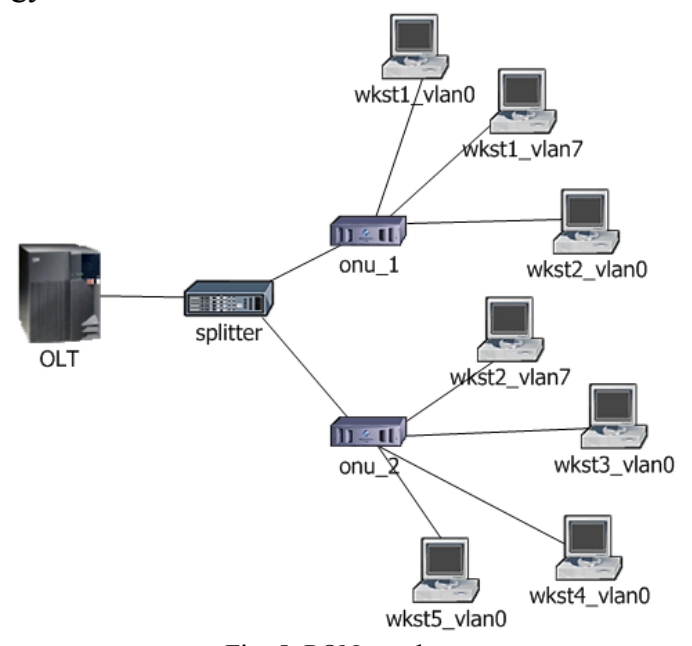

Fig. 5. PON topology 
1) $O L T$

The primary task of OLT is to calculate the size of the grant for each ONU unit in each cycle. The number of units is manually set. The size of grants allocated is calculated either statically or dynamically. In static mode, the same grant's size is set for all ONUs. In dynamic mode, the size is calculated on the basis of requests sent by ONUs. The physical address of OLT is set to 99 . The OLT may connect up to eight terminal units $[21,22]$.

2) Splitter

Passive optical splitter is intended to simulate the behavior of such a device in practice. The splitter is receiving the packets on point-to-point interfaces and forwarding them via all point-to-point transmitters to the destination node. This procedure is simulated by two processor blocks: proc_tx and proc_rx. The processors contain simple process models where only two states init and receive_send take care about the functionality of the splitter. The splitter is designed to provide a split ratio of $1: 8$, but can be easily expanded to $1: N$. The splitter itself is generating a certain traffic delay. This is due to the calculations for copying the packet to the individual transmitters. It is necessary to consider this delay when the attenuation for specific connection between OLT and splitter is calculated. The delay is in the range of 1 to 4 microseconds. The value depends on current data stream [21].

3) $\mathrm{ONU}$

ONU receives all data sent from the OLT. Based on the data type and their addresses, the ONU will decide whether they are going to be processed or discarded/forwarded. In the developed simulation model, ONU receives only the GATE messages which inform about the start time of the grant and its length. It is needed to set the attributes of the physical address for each ONU. The ONU model includes eight queues. Each queue processes received data within defined priority (weight), see the parameters for all traffic classes in TABLE I. Data classification is performed based on different point-to-point transmitters/receivers marked as eth_rx_vlanX or eth_tx_vlanX. In practice, this classification procedure is ensured by a specialized device (packet classifier) or the VLANs can be used [21].

TABLE I

TRAFFIC CLASS FOR EACH QUEUE

\begin{tabular}{|l|l|l|}
\hline Queue & \multicolumn{1}{l|}{ Weight } & Type traffic \\
\hline queue 0 & $\mathbf{0 . 3 0}$ & real-time services - telephony and video-tele. \\
queue 1 & $\mathbf{0 . 2 8}$ & real-time services - video streaming \\
queue 2 & $\mathbf{0 . 1 1}$ & signalization \\
queue 3 & $\mathbf{0 . 0 9}$ & network management \\
queue 4 & $\mathbf{0 . 0 7}$ & critical data \\
queue 5 & $\mathbf{0 . 0 6}$ & critical data \\
queue 6 & $\mathbf{0 . 0 5}$ & best-effort \\
queue 7 & $\mathbf{0 . 0 4}$ & less important services \\
\hline
\end{tabular}

The TABLE II shows the basic set of simulation parameters. The length of one cycle was set to $188 \mu$ s. This time is the tradeoff between the cycle length that can handle larger amounts of data and low delay. The data rate of all links in the network was set to 1 Gbps which is in accordance with the standard EPON. In order to provide higher simplicity, optical links generate no delay. BER (Bit Error Rate) for all simulated optical fibers was set to $10^{-10}$ which is one of the typical values.
TABLE II

NETWORK PARAMETERS IN THE SIMULATION

\begin{tabular}{|l|l|}
\hline Parameter & Value \\
\hline Number of ONU & $\mathbf{2}$ \\
Transmission speed of optical link & $\mathbf{1 ~ G b p s}$ \\
Transmission speed of LAN & $\mathbf{1 ~ G b p s}$ \\
Optical delay of line & $\mathbf{0 ~ s}$ \\
BER of optical fiber & $\mathbf{1 0}$ \\
Number of classes for QoS & $\mathbf{8}$ \\
Size of cycle & $\mathbf{1 8 8} \boldsymbol{\mu \mathbf { s }}$ \\
Guard interval between grants & $\mathbf{1} \boldsymbol{\mu s}$ \\
Guard interval between cycles & $\mathbf{7} \boldsymbol{\mu s}$ \\
\hline
\end{tabular}

\section{B. Simulation of TCP/IP Network}

As an alternative to the passive optical network, classic TCP/IP network has been selected. This type of network forms the platform for the vast majority of current networks, hence it is also a good candidate for a hybrid multimedia network. Low costs for deployment and operation are the main advantage of TCP/IP- based networks. Moreover, it is a reliable and well recognized communication platform.

The goal of performed simulation was to identify the network parameters that have the greatest impact on the performance of multimedia network operated over TCP/IP platform. Another task was to find optimal settings of these parameters. A side target was to define some recommendations to achieve the best network KPIs.

In more detail, the influence of the selected routing protocol, the implementation of QoS mechanism and the level of core links utilization were analyzed. As the output, the network characteristics (such as end-to-end delay, jitter and throughput) were discussed. The topology of developed simulation model is shown in Fig. 6.

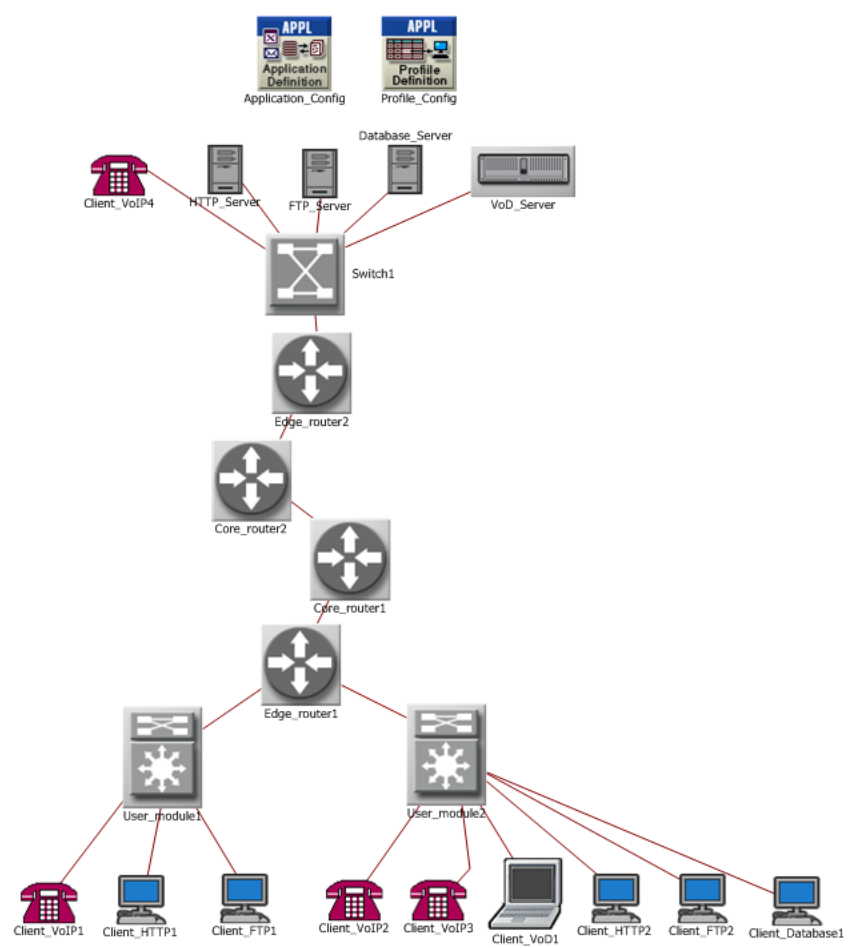

Fig. 6. TCP/IP based hybrid multimedia network

The backbone of the designed topology is composed of four routers (two edge routers and two core routers) that simulate general WAN (Wide Area Network) to provide the connections between user workstations and application 
servers. The end terminals are connected to the access network via the subscriber module, whose task is to process incoming or outgoing calls. In addition, this module also provides a functionality of standard switch.

The structure of applications and services operated in current multimedia networks is very variable, so to provide highly realistic scenario, the simulation model defines the following applications:

- $\quad$ voice calls (VoIP),

- videoconferencing,

- web browsing (HTTP),

- database access (SOAP),

- $\quad$ file transfer (FTP).

All network traffic is started in 11 seconds after the beginning of simulation. This time offset is long enough to complete all initial configuration processes that could affect the simulation results. Separate profile that defines the amount of data generated and the time characteristics, was defined for each network application. The entire simulation took 10 minutes which is sufficient time to recognize the required network characteristics.

\section{ANALYSIS OF SIMULATION RESULTS}

As already mentioned in the previous chapter, the simulation of hybrid multimedia network was divided into two parts PON and TCP/IP.

\section{A. Discussion on Simulation Results - PON}

The goal of the first part of performed simulation was to evaluate the delay of transmitted data and throughput in the designed passive optical network. Because of the high computational demands, the simulation time was set to $0.04 \mathrm{~s}$ and data traffic has been generated by the stations for 0.01 seconds from the beginning of the simulation. Despite such a short simulation time, this setting is sufficient for the required verification of monitored parameters.

Fig. 7 shows the throughput of the formed network, respectively throughput between the ONU and a client station. The graph shows that optical network is utilized at $100 \%$ as the line speed was set to $1 \mathrm{Gbps}$.

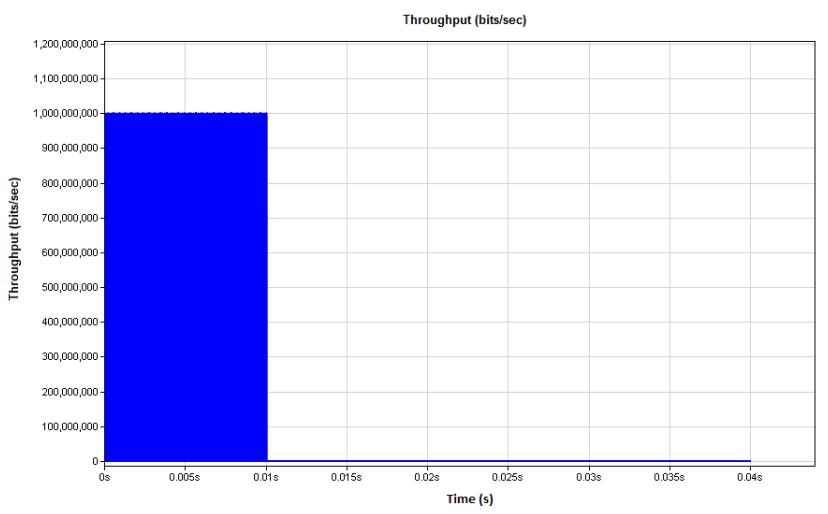

Fig. 7. Throughput between ONU and the client station

The communication delay for data stream between the client and OLT is shown in Fig. 8. The client station generates the traffic for simulation time of 0.01 seconds. The largest delay contributors are the splitter and ONU. The splitter spends certain time by copying all incoming packets and forwarding them to all transmitters. ONU has to process each packet and decide about the subsequent procedure (forward/drop). On the other hand, the optical link's contribution is very low.

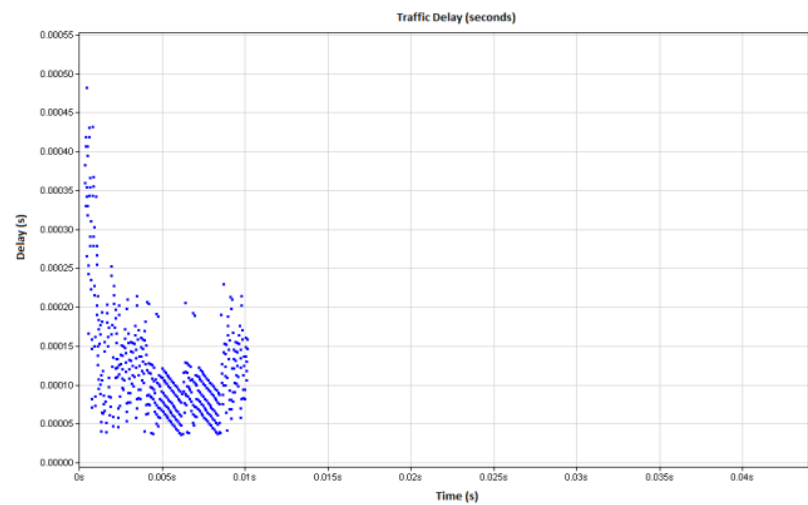

Fig. 8. Traffic delay

The simulation of passive optical network can be summarized so that this communication platform provides very low end-to-end delay, even for maximal utilization of network link. Therefore, it can be said that PON is suitable for the transmission of multimedia data that have strict requirements on delay and jitter.

Despite the very good results obtained from the simulations, PON has several significant drawbacks:

- High deployment costs - comparing to IP networks, the costs of construction and operation are much higher.

- Security (wiretap) - all wavelengths are propagated to all end units which can be tapped for the attacker. Therefore, it is necessary to implement the encryption method.

- Relatively high attenuation - attenuation caused by passive optical splitters in the distribution network.

\section{B. Discussion on Simulation Results - TCP/IP}

In order to clearly present the influence of various mechanisms on network performance, the generated traffic is set in the way that the core-network links are utilized by $80 \%$ in average. It is generally understood that in lightly loaded networks it is not necessary to solve performance analyzes or implement additional mechanisms (e.g. QoS), as the implementation of these mechanisms has practically no impact.

1) Analysis of routing protocols

The influence of routing protocols on the network traffic characteristics was analyzed as the first. We have developed two completely identical scenarios (see Fig. 6) where the routing protocol (RIP or OSPF) was the only difference. The obtained results clearly showed that even though OSPF generates higher traffic overhead and greater procedural delays within the network nodes (see Fig. 9), it achieves significantly better results in the case of high-loaded network. It is mostly because it can better solve the network congestion issue by the integrated network load balancing mechanism and faster convergence. So, the OSPF allows achieving higher throughput and lower delay for network services.

Fig. 10 shows the progress of the total download time for the 10MB file from the FTP server to the client station. The graph clearly shows, that in case of RIP, the download time is increasing almost linearly as the network load is growing. 
On the contrary, the OSPF routing protocol can provide almost constant download response time even at high network load.

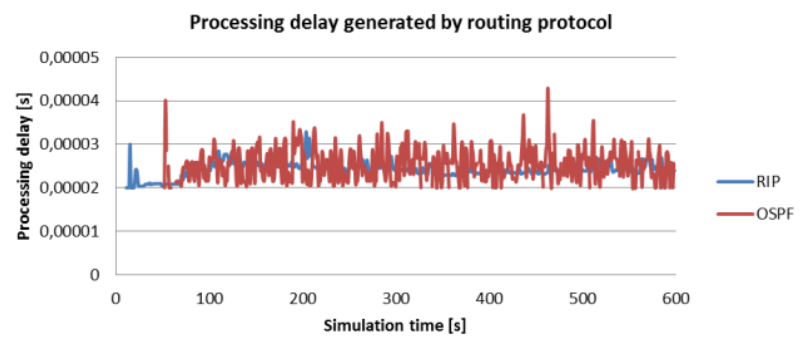

Fig. 9. Processing delay generated by routing protocols on Core_router2

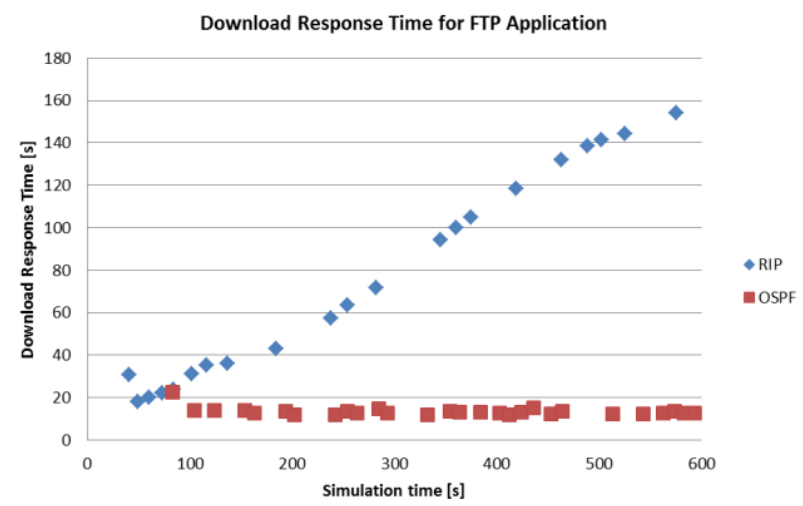

Fig. 10. Download response time for FTP application on Client_FTP1

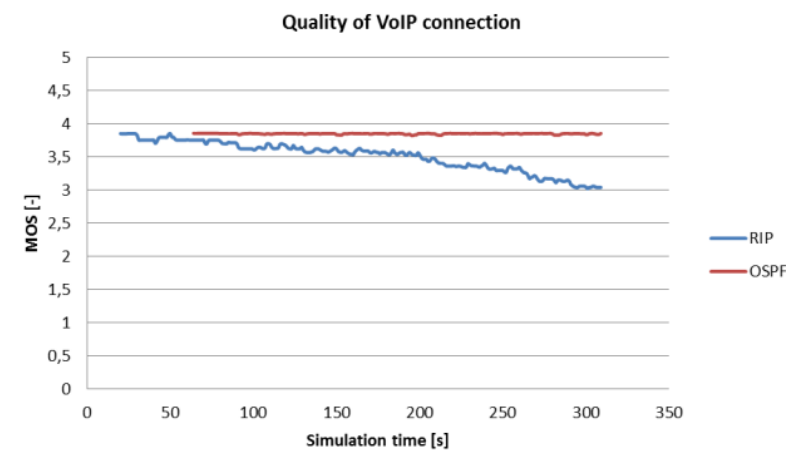

Fig. 11. Call quality between terminals Client_VoIP3 and Client_VoIP4

The OSPF protocol also provides better results for VoIP service. Fig. 11 shows the MOS (Mean Opinion Score) based quality evaluation of VoIP call between the VoIP terminals Client_VoIP3 and Client_VoIP4. The RIP scenario provides evident quality degradation when the network load is high. On the other hand, OSPF can maintain the connection quality at a very stable level. The maximal value MOS value of 3.85 corresponds to a very good quality considering used codec G.729.

2) Analysis of QoS Mechanism

Although the OSPF clearly showed better performance compared to the RIP protocol, to meet the strict requirements of real-time traffic (voice, video), the network extension by QoS mechanisms is needed. This implementation requirement brings certain contribution only for highly loaded networks for sure.

Basic scenario with OSPF protocol was duplicated and the DiffServ mechanism was implemented into this new scenario. Fig. 12 shows the end-to-end delay for VoIP call realized between the VoIP_Client 3 and VoIP_Client 4 . In the RIP scenario, the E2E delay is increasing rapidly and approximately in the middle of the call reaches a critical value of $150 \mathrm{~ms}$ for which the end-user quality is very degraded. Among scenarios, OSPF and OSPF + QoS is not so noticeably different, but with closer examination was found out that the implementation of QoS mechanisms to achieve greater stability values of delay and jitter value is for scenarios with lower QoS mechanism, which is very important for the realization of high-quality multimedia transmissions in real-time.

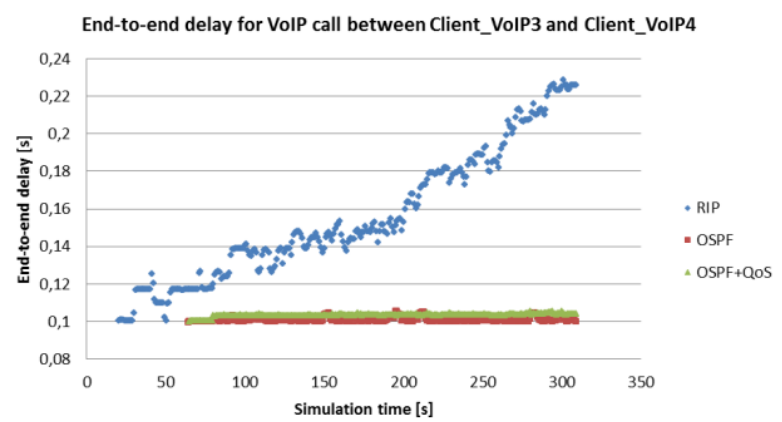

Fig. 12. E2E delay for VoIP call between VoIP_Client3 and VoIP_Client4

Implemented QoS mechanism does not have a favorable effect only on E2E delay or jitter, but also on the overall network throughput. With the classification process and separate treatment of each traffic class according to their requirements, the network resources are efficiently utilized. This prevents the cumulative burden of network nodes and links from retransmissions of packets dropped inside the congested routers.

This phenomenon is clearly shown in Fig. 13, where the utilization of the link between the routers Core_Router1 and Core_Router 2 is depicted. In case of the RIP scenario, all available network resources are exhausted which affects performance of all services. In contrast, DiffServ technology most effectively manages network resources and allows keeping the link utilization below $80 \%$.

\section{CONCLUSION}

In order to define appropriate communication technology for hybrid multimedia network, a routing protocol and any other supporting mechanisms ensuring the required quality of service, the performance analysis was carried out in a simulation environment OPNET Modeler.

Two communication technologies - passive optical network and classic TCP / IP network were evaluated. Even though both these technologies are quite different and cannot be directly compared, the emphasis was put on the identification of the key network elements or parameters that have the greatest effect on the basic QoS characteristics like E2E delay, jitter and throughput.

Moreover, in case of TCP/IP network, the influence of routing protocol and implementation of QoS mechanism on the overall network efficiency has been studied. Achieved simulation results and their analyses have been discussed in the previous chapter.

Overall, the performed analysis can be summed up so the proper choice of the routing subsystem for multimedia networks is influenced by many factors. It is possible to define a set of core network elements and parameters/subsystems that contribute to the overall network 
performance the most, but, at the same time, it is clear that particular network topology, level of network load and composition of operated applications have to be taken into account. Moreover, the total deployment and maintenance costs play a significant role.

\section{REFERENCES}

[1] Kalyan, G., P., S., Prasad, D., V., V.: Optimal selection of Dynamic Routing Protocol with real time case studies. 2012 International Conference on Recent Advances in Computing and Software Systems [online]. IEEE, 2012, s. 219-223 [cit. 2014-01-13]. DOI: 10.1109/RACSS.2012.6212727. Available on: http://goo.gl/Rm3LGM.

[2] Thorenoor, S. G. (2010). Dynamic Routing Protocol Implementation Decision between EIGRP, OSPF and RIP Based on Technical Background Using OPNET Modeler. 2010 Second International Conference on Computer and Network Technology, 191-195. doi:10.1109/ICCNT.2010.66.

[3] Hendrick, C. (1988). Routing Information Protocol - RFC 1058, 1-33. URL: http://tools.ietf.org/rfc/rfc1058.txt.

[4] Malkin, G. (1998). RIP Version 2 - RFC 2453, 1-40. URL: http://tools.ietf.org/rfc/rfc2453.txt.

[5] Fitigau, I.; Toderean, G., "Network performance evaluation for RIP, OSPF and EIGRP routing protocols," International Conference on Electronics, Computers and Artificial Intelligence (ECAI), 2013 , vol. 1, no. 1, pp.1-4, 27-29 June 2013.

[6] Moy, J. (1989). OSPF - RFC 1131, 1-107. URL: http://tools.ietf.org/pdf/rfc1131.pdf.

[7] Moy, J. (1998). OSPF Version 2 - RFC 2328, 1-245. URL: http://tools.ietf.org/pdf/rfc2328.pdf.

[8] Colton, R., Ferguson, D., Moy, J. (1999). OSPF for IPv6 - RFC 2740, 1-93. URL: https://tools.ietf.org/rfc/rfc5340.txt.

[9] Lan, L., Li, L., \& Jianya, C. (2012). A Multipath Routing Algorithm Based on OSPF Routing Protocol. 2012 Eighth International Conference on Semantics, Knowledge and Grids, 269-272. doi:10.1109/SKG.2012.7.

[10] Szigeti, T., Hattingh, C. End-to-End QoS Network Design: Quality of Service in LANs, WANs, and VPNs. Cisco Press, 2004.

[11] Hosek, J. Nové metody zajištění kvality služeb v datových sítích: doktorská práce. Brno: Vysoké učení technické v Brně, Fakulta elektrotechniky a komunikačních technologií, Ústav telekomunikací, 159 s., 2011.

[12] Park, I. K. QoS in Packet Networks. Springer, 2005.

[13] Velte, J. T. Cisco: A Beginner's Guide. McGraw-Hill, 2001

[14] Flannagan, M. E. Administering Cisco QoS for IP Networks. Syngress Publishing, 2001.

[15] Blake, S., Black, D., Carlson, M., Davies, E., Wang, Z., Weiss, W.: An Architecture for Differentiated Service, RFC2475, 1998.

[16] Marchese, M. QoS Over Heterogeneous Networks. Wiley Publishing, 2007. ISBN 9780470017524.

[17] Cisco Systems Inc. Catalyst 4500 Series Switch Cisco IOS Software Configuration Guide [online]. 2004. Available on: http://www.cisco.com/en/US/docs/switches/lan/catalyst4500/12.2/25e w/configuration/guide/OL_6696.pdf.

[18] Cisco Systems Inc. QoS: Classification Configuration Guide, Cisco IOS XE Release 3S [online]. 2013. Available on: http://goo.gl/VCKRHy.

[19] Cisco Systems Inc. Inter-Switch Link and IEEE 802.1Q Frame Format [online]. 2006. Available on: http://www.cisco.com/image/gif/paws/17056/741 4.pdf.

[20] Sifta, R., Munster, P., Krajsa, O., Filka, M. Simulation of bidirectional traffic in WDM- PON networks. Przeglad Elektrotechniczny, 2014, roč. 90, č. 1, s. 95-100. ISSN: 0033- 2097.

[21] Lafata, P., Vodrazka J. Pasivní optická sít' GPON. Access server [online]. 23.05. 2009, č. 2009050002. ISSN 1214-9675. Available on: http://access.feld.cvut.cz/view.php?cisloclanku=2009050002.

[22] ITU-T: G.983.1 - Broadband optical access systems based on Passive Optical Networks (PON). [online], Internet: http://www.itu.int/rec/TREC-G.983.1-200501-I/. ITU-T, January 2005. 